Goldschmidt 2021 Abstract

https://doi.org/10.7185/gold2021.6001

\section{Nano- and mesoscale structures and chemical labelling support nanoscale assembly of amorphous calcium carbonate from prenucleation clusters}

\author{
STEPHAN E. WOLF ${ }^{1}$, BRUNO COLAS ${ }^{2}$, SIMON CLARK ${ }^{2}$, \\ DORRIT JACOB ${ }^{3}$, DIRK ZAHN ${ }^{1}$, SHIFI KABABYA ${ }^{4}$, \\ ASHER SCHMIDT ${ }^{4}$, PATRICK DUCHSTEIN ${ }^{1}$, PHILIPP \\ SCHODDER $^{1}$ AND VITALIY PIPICH ${ }^{5}$ \\ ${ }^{1}$ Friedrich-Alexander University Erlangen-Nürnberg (FAU) \\ ${ }^{2}$ Macquarie University \\ ${ }^{3}$ Australian National University \\ ${ }^{4}$ Technion \\ ${ }^{5}$ Forschungszentrum Jülich $\mathrm{GmbH}$ \\ Presenting Author: stephan.e.wolf@fau.de
}

Transient amorphous calcium carbonate (ACC) is a crucial precursor in various mineralizing environments, and insight into its ultrastructure is vital for understanding formation pathways and identifying molecular control mechanisms by additives. Here, we provide a detailed model for ACC at the atomic level received by applying Monte Carlo simulations constrained by both X-ray and neutron scattering data. This ultrastructural study reveals that synthetic ACC is composed of approx. twonanometer-sized units with a high degree of near-range order. Small-angle neutron scattering experiments evidence a hierarchical structure indicative of a multi-step self-assembly. Molecular dynamics simulation corroborates that ACC is akin to a glassy gel, while high-resolution transmission electron microscopy shows that the domain-structure impacts ACC crystallization. These findings support that ACC forms by a nanoparticle aggregation process starting from solute species that are only a couple of nanometers in size, i.e., prenucleation clusters (PNC). To corroborate the involvement of PNCs, we took a close look at small-molecular-weight additives that strongly differ in their individual impact on the formation of $\mathrm{CaCO}_{3}$ and stabilization of ACC. We find that only those additives effectively impede precipitation if they actively trigger PNC formation. According to simulations, these additives incorporate into PNCs, over-stoichiometrically enhancing $\mathrm{CaCO}_{3}$ solubility as determined by in-situ Ca-activity measurements. Moreover, they co-precipitate with ACC, as confirmed by solidstate NMR, and retard ACC crystallization in dry and wet environments. In short, additives selectively co-precipitate with ACC only when they strongly interact with PNCs; additives that do not strongly interact with PNCs in solution can hardly be traced in the ACC precipitate. Also, these latter additives hardly impact ACC formation and stability. This points at specific molecular interaction between PNCs and additives that allow chemical labelling of PNCs and corroborates that ACC forms via PNC aggregation.

Thus, our results suggest that ACC forms via aggregation of PNCs, i.e., complex calcium carbonate ion associates, and that this formation process is susceptible to additives that molecularly interact with PNCs. These results motivate us to re-assess the role of small molecules in biomineralization and geochemistry to re-consider ACC formation as an aggregation process of complex solute species.

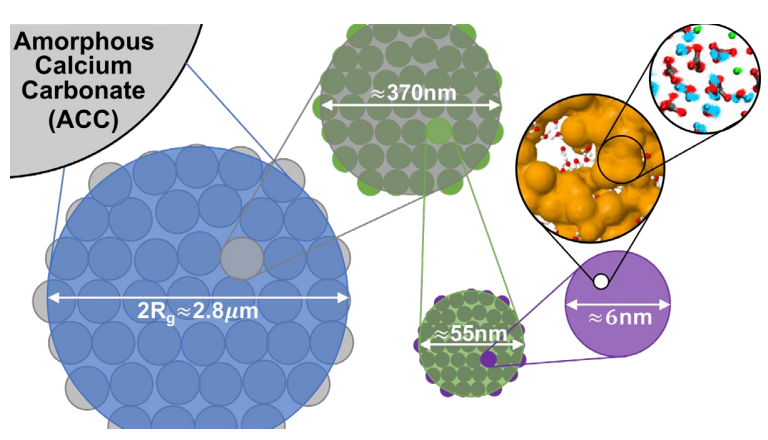

